\title{
Estudio de la carga externa e interna de fósforo y aplicación de modelos empíricos de eutrofización en las aguas de la Albufera de Adra
}

\author{
de Vicente, I. \& L. Cruz-Pizarro \\ Instituto del Agua. Universidad de Granada. C/ Ramón y Cajal, 4. 18071 Granada. Spain. \\ ivicente@ugr.es
}

\section{RESUMEN}

Los resultados de la aplicación de un balance de masas de fósforo $(\mathrm{P})$ realizado en los dos sistema lagunares más importantes de las Albuferas de Adra, revelan el papel fundamental que juega el sedimento en el ciclo de dicho nutriente. Sobre una base anual, el sedimento de ambas lagunas se comporta como un "sumidero" de P (-168.5 y -29.7 kg TP retienen la laguna Honda y la laguna Nueva, respectivamente).

En éste trabajo se han ensayado además, cuatro modelos empíricos de masa de agua (Vollenweider, 1976; Dillon \& Rigler, 1974; OCDE, 1982; Foy, 1992) para la predicción de la concentración promedio anual de fósforo total (TP) en las lagunas (en dos años consecutivos) a partir de variables como la carga hidráulica anual y la carga externa anual de fósforo. Los resultados obtenidos muestran que, en todos los casos, la simulación proporciona subestimas de la concentración de TP medida en el sistema, en un rango comprendido entre el 39 y el $75 \%$ en la laguna Honda y entre el 9 y el 33\%, en la laguna Nueva.

Por último, cabe destacar la elevada carga externa anual de $\mathrm{P}$ que llega a la laguna Honda, muy superior al valor tolerable (crítico) estimado según los modelos de carga crítica ensayados.

Palabras clave: Fósforo, carga externa, carga interna, balance, eutrofización, lagunas litorales.

\begin{abstract}
The results of the use of a mass balance for phosphorus $(P)$ made in the two more important coastal lagoons of Albufera de Adra, reveal the fundamental role that the sediment plays in the cycle of this nutrient. On a year-to-year basis, the sediment of both lagoons can be considered as a "sink" for P (-168.5 and-29.7 kg of TP are retained by laguna Honda and laguna Nueva, respectively).

In this job four empiricals models of water mass have also been tested (Vollenweider, 1976; Dillon \& Rigler, 1974; OCDE, 1982; Foy, 1992) for the prediction of the phosphorus annual mean concentration (TP) in the lagoons (in two consecutive years) using variables such as annual hydraulic and annual external phosphorus loading values. The results show that the simulation values underestimate the TP concentrations measured in the system, within a range between 39 and $75 \%$ for Honda lagoon, and between 9 and 33\% un Nueva lagoon.

Finally, it is important to remark the elevated P external load that enters Honda lagoon, much higher than the tolerable value (critical) estimated by the critical loading models tested.
\end{abstract}

Keywords: Phosphorus, external load, internal load, balance, eutrophication, coastal lagoons.

\section{INTRODUCCIÓN}

De entre los nutrientes, el fósforo $(\mathrm{P})$ es considerado como el limitante por excelencia de la productividad fitoplanctónica (Reynolds, 1984; Lampert \& Sommer, 1997; García-Ruiz et al., 2001). Por ello, la concentración de fósforo total (TP) ha sido tradicionalmente empleada como índice de la calidad del agua y del estado trófico de los ecosistemas acuáticos. Como quiera que la cantidad de TP en la columna de agua depende de los aportes alóctonos al sistema (carga externa), de la exportación a través de los efluentes, de los procesos de sedimentación y de los de liberación desde los sedimentos (carga interna), la identificación y cuantificación de las cargas externa e interna de $\mathrm{P}$ al sistema se revela como un aspecto básico esen- 
cial sobre el que adoptar medidas de prevención y/o planificar futuros esfuerzos de control de la calidad de las aguas.

Mientras que la cuantificación de la carga externa de P no presenta generalmente notables dificultades, la evaluación de la carga interna requiere de un mayor esfuerzo y su estudio puede llevarse a cabo bajo diferentes aproximaciones. Entre ellas, el método más directo y adecuado consiste en la aplicación del balance de masas de P (Søndergaard, 1989), una metodología que permite conocer la retención neta de $\mathrm{P}$ durante un determinado período de tiempo, aportando información acerca de los posibles procesos involucrados en la retención y liberación de $\mathrm{P}$ desde los sedimentos. Así mismo, el hecho de que los nutrientes presentes en la interfase agua-sedimento se encuentren en equilibrio dinámico entre la fase sólida (sedimento) y la líquida (agua intersticial), hace que la retención o liberación de $\mathrm{P}$ desde los sedimentos dependa en última instancia de la posición de este equilibrio, caracterizándose por tanto, la carga interna por una extraordinaria variabilidad tanto estacional como interanual (Golterman, 1995; Calzada-Bujak et al., 2001).

La eutrofización de los ecosistemas acuáticos representa actualmente uno de los principales problemas que afectan a la calidad del agua (Ryding \& Rast, 1992), por lo que existe una necesidad creciente en la aplicación de modelos simples de eutrofización de lagos y embalses que traten de predecir la concentración de $\mathrm{P}$ en el ecosistema acuático a partir de la carga externa de P que recibe (Sakamoto ,1966; Dillon \& Rigler, 1974; Oglesby \& Schafner, 1978; Rast \& Lee,1978; Foy, 1992). Éstos modelos representan herramientas extraordinariamente útiles tanto para la gestión como para la investigación del problema de la eutrofización (Ahlgren et al., 1988). Además proporcionan una visión más integrada de la realidad, al considerar al ecosistema acuático y a su cuenca de captación como una unidad, de modo que las actividades que se desarrollen en la cuenca, y que en última instancia afectarán a la carga externa de $\mathrm{P}$, repercutirán en la calidad del agua del sistema receptor (Kalff, 2002). De todos ellos, seguramente el más conocido y todavía hoy más utilizado, es el que se conoce como modelo de Vollenweider (1968).

Los objetivos de este trabajo han sido:

a) Estima de la carga externa e interna de $\mathrm{P}$ al sistema mediante la aplicación de un balance de masas de TP.

b) Aplicación de modelos de masa de agua y de carga crítica en los sistemas de estudio.

\section{ÁREA DE ESTUDIO}

La Albufera de Adra, considerada como la zona húmeda natural más importante del sureste peninsular, está compuesta por dos lagunas costeras someras: laguna Honda y laguna Nueva. Algunas de las principales características limnológicas de las lagunas así como los resultados de un estudio sobre la evolución del estado trófico y de la identificación del nutriente limitante de la producción algal se recogen en CruzPizarro et al. (2003).

El aporte superficial de agua a las lagunas procede del río Adra, a través de la Acequia Real de Adra que desemboca en ambas lagunas tras bifurcarse poco antes de llegar a las mismas. La laguna Honda recibe la descarga de una sóla acequia de riego mientras que en la laguna Nueva vierten dos acequias.

La laguna Honda recibe, además, la descarga directa de tres ramblas: "La Estanquera", "Las Adelfas" y "Del Alto", que drenan una cuenca vertiente de $13.7 \mathrm{~km}^{2}$ (Fuente: Mapa Topográfico de Andalucía, hoja 1057 3-2). Los tres cauces drenan la vertiente Sur de Sierra Alhamilla y las estribaciones más suroccidentales de la Sierra de Gádor, dirigiéndose en dirección norte-sur al área de la Albufera. Estos cauces permanecen secos todo el año, exceptuando los momentos de lluvias torrenciales, en los cuales puede producirse la entrada de gran cantidad de materia en suspensión a las lagunas. De hecho, en los últimos años (especialmente desde 
las lluvias acontecidas a finales de Diciembre de 2000) se ha observado el crecimiento de un notable cono de deyección en la parte central (estrecho) de la laguna Honda, lo que podría dar lugar a una indeseable división de la laguna en dos cubetas independientes.

Es necesario indicar la existencia de un trabajo preliminar sobre el balance másico de $\mathrm{P}$ en las lagunas de la Albufera de Adra (de Vicente et al.,2001).

\section{MATERIAL Y MÉTODOS}

El período de estudio estuvo comprendido entre Julio de 1999 y Junio de 2001. La toma de muestras, tanto de la columna de agua como de las acequias que vierten en las lagunas, se llevó a cabo con una periodicidad básica, aproximadamente quincenal. Para la obtención de muestras de la zona pelágica se seleccionaron dos estaciones localizadas en la zona de máxima profundidad de cada una de las lagunas, de acuerdo con el mapa batimétrico realizado por Cruz-Pizarro et al. (1992). En cada una de ellas se procedió a la toma de muestras a tres profundidades en el perfil vertical $(10 \mathrm{~cm}$, $z_{\text {max }} / 2$ y $z_{\max }$ ).

La concentración de fósforo total (TP) se determinó siguiendo el método "azul de molibdeno" (Murphy \& Riley, 1962), previa digestión de la muestra con persulfato potásico. Los valores que se muestran en el apartado de Resultados y Discusión corresponden a la media aritmética de la concentración de TP en las 3 profundidades muestreadas.

El índice de saturación en diferentes minerales se ha obtenido mediante la aplicación del programa informático Hydrowin (versión 3.0), a partir de los datos de alcalinidad, cationes y aniones mayoritarios así como de variables físico-químicas medidas in situ, tales como el $\mathrm{pH}$, la Conductividad y la Temperatura.

- Las variables físico-químicas (T, pH y Conductividad) se midieron mediante una sonda multiparamétrica TURO (mod. T-611).
- La alcalinidad total del agua se determinó mediante la valoración automática de la muestra con $\mathrm{HCl}$ (titriador METROHM 716 DMS).

- La determinación de los cationes $\left(\mathrm{Na}^{+}, \mathrm{K}^{+}\right.$, $\mathrm{Ca}^{+2}$ y $\left.\mathrm{Mg}^{+2}\right)$ y aniones mayoritarios $\left(\mathrm{Cl}^{-}\right.$, $\mathrm{SO}_{4}{ }^{-2}$ ) se ha realizado mediante cromatografía iónica en un DIONEX (DX 300), previa filtración de la muestra a través de filtros de $0.2 \mu \mathrm{m}$.

Los datos sobre la velocidad máxima del viento han sido proporcionados por la estación meteorológica instalada por la Consejería de Medio Ambiente en las afueras del pueblo de Adra.

Para la determinación de la composición granulométrica del sedimento superficial de ambas lagunas se ha seguido el método de la "pipeta de Robinson" (Robinson, 1922), basado en la separación de las partículas minerales en función de su diferente velocidad de sedimentación.

\section{Balance másico de fósforo}

La ecuación general del balance de masas para una sustancia es:

$$
\frac{\mathrm{dM}_{\mathrm{x}}}{\mathrm{dt}}=\mathrm{L}_{\mathrm{ext}}-\mathrm{S} \pm \mathrm{L}_{\mathrm{int}}
$$

donde, $\mathrm{M}_{\mathrm{x}}=$ masa de TP en la columna de agua, $\mathrm{L}_{\text {ext }}=$ carga externa de TP al sistema, $\mathrm{S}=$ salida superficial de TP del sistema y $\mathrm{L}_{\text {int }}=$ carga interna de TP al sistema (incluidos los aportes/ descargas subterráneas).

En el caso de las lagunas de la Albufera de Adra, la inexistencia de salidas superficiales desde el sistema, permite la simplificación de la ecuación general a la siguiente expresión:

$$
\mathrm{L}_{\text {int }}=-\mathrm{L}_{\text {ext }} \pm \Delta \mathrm{TP}
$$

Si $\mathrm{L}_{\text {int }}<0$ predominan los procesos de retención de TP en los sedimentos, mientras que si $\mathrm{L}_{\text {int }}>0$ son los procesos de liberación de TP los que predominan. 
Tabla 1. Modelos empíricos que predicen la concentración promedio anual de TP $\left(\mathrm{P}_{\mathrm{c}}, \mu \mathrm{g} / 1\right)$. Empirical models to predict the annual mean TP concentration $\left(P_{c}, \mu g / l\right)$.

\begin{tabular}{ll}
$\mathrm{P}_{\mathrm{c}}=\left(\mathrm{L}_{\text {ext }} / \mathrm{q}_{\mathrm{s}}\right)(1-\mathrm{R})$ & Dillon \& Rigler $(1974)$ \\
$\mathrm{P}_{\mathrm{c}}=\left(\mathrm{L}_{\text {ext }} / \mathrm{q}_{\mathrm{s}}\right) /\left(1+\sqrt{\mathrm{t}_{\mathrm{w}}}\right)$ & Vollenweider $(1976)$ \\
$\mathrm{P}_{\mathrm{c}}=1.55\left(\mathrm{P}_{\mathrm{in}} /\left(1+\sqrt{\mathrm{t}_{\mathrm{w}}}\right)\right)^{0.82}$ & OCDE $(1982)$. \\
$\mathrm{P}_{\mathrm{c}}=\left(1.118 \mathrm{P}_{\mathrm{in}}\right) /\left(1+\sqrt{\mathrm{t}_{\mathrm{w}}}\right)^{1.135}$ & Fórmula general. \\
\hline
\end{tabular}

\section{Cuantificación de la carga externa de P}

\section{1) Deposición atmosférica $\left(\mathrm{L}_{\mathrm{ext} 1}\right)$}

El cálculo de la deposición atmosférica de $\mathrm{P}$ requiere la elección del coeficiente más adecuado al sistema de estudio, así como el conocimiento del área superficial de la masa de agua. En el caso que nos ocupa, como quiera que el terreno circundante se clasificaría dentro de la categoría rural agrícola, se le ha asignado un coeficiente de exportación de $0.66 \mathrm{~kg} \mathrm{P} \mathrm{ha}^{-1} \mathrm{año}^{-1}$ (Hendry et al., 1981), y conocida la extensión superficial de cada laguna la deposición atmosférica media anual de $\mathrm{P}$ se podría estimar en 5.28 y $17.19 \mathrm{~kg}$ P para la laguna Honda y la laguna Nueva, respectivamente.

\section{2) Carga puntual ( $\left.\mathrm{L}_{\mathrm{ext} 2}\right)$}

La medición del caudal de agua que vierte en las lagunas a través de la red de acequias así como la determinación de la concentración de TP en la misma, ha permitido la cuantificación de la carga externa puntual de TP a las lagunas estudiadas.

La medida del caudal se ha realizado mediante aforo químico (empleando $\mathrm{NaCl}$ como marcador) así como mediante la utilización de "objetos inertes" a la deriva. Una vez calibrados, se ha optado, en la mayoría de las ocasiones, por emplear el segundo de ellos.

\section{3) Carga difusa ( $\left.\mathrm{L}_{\mathrm{ext}} 3\right)$}

Uno de los métodos más usualmente empleado para la estima de las cargas difusas externas es la utilización de las "unidades de carga por superficie" basadas en el uso, a su vez, de coeficientes de exportación de nutrientes. Este proce-
Tabla 2. Modelos de carga crítica de fósforo $\left(\mathrm{L}_{\mathrm{c}}, \mathrm{mg} \mathrm{m}^{-2} \mathrm{año}^{-1}\right)$. Models used to quantify the critical loading values $\left(L_{c}, \mathrm{mg} \mathrm{m}^{-2}\right.$ year-1).
$\mathrm{L}_{\mathrm{c}}=20 \cdot \mathrm{q}_{\mathrm{s}} \cdot\left(1+\sqrt{\mathrm{t}_{\mathrm{w}}}\right)$
OCDE (1982)
$\mathrm{L}_{\mathrm{c}}=17\left(\overline{\mathrm{z}} / \mathrm{t}_{\mathrm{w}}\right)^{0,6} \cdot \overline{\mathrm{Z}}^{0,4}$
Vollenweider (1976)

dimiento se basa en la observación de que, en condiciones hidrológicas medias, en una cuenca a lo largo del ciclo anual, una determinada actividad del uso del terreno exportará una carga relativamente constante de nutrientes por unidad de superficie a las aguas receptoras que drenan en dicho terreno (Ryding \& Rast, 1992).

La carga externa de P que recibe la laguna Honda a través de las ramblas se ha estimado mediante la delimitación de la cuenca vertiente (Mapa Topográfico de Andalucía; hoja 1057 32), así como la identificación de la extensión ocupada por los diferentes usos del suelo $\left(\mathrm{A}_{\mathrm{i}}\right.$, ha) y de la litología predominante. La elección del coeficiente de exportación de P (C.E., kg P $\mathrm{ha}^{-1}$ año $\left.{ }^{-1}\right)$ para cada uso del terreno se ha realizado teniendo en cuenta las categorías descritas por Sonzogni et al. (1980).

Finalmente, la carga externa difusa de $\mathrm{P}$ ( $\mathrm{L}_{\text {ext }}, \mathrm{kg} \mathrm{TP}$ ) se ha estimado como:

$$
\mathrm{L}_{\text {ext } 3}=\sum\left(\mathrm{A}_{\mathrm{i}} \cdot \mathrm{CE}_{\mathrm{i}}\right)
$$

\section{Aplicación de Modelos de Eutrofización y de Carga crítica}

Dada la extraordinaria variabilidad interanual observada, se ha optado por el estudio comparativo de dos períodos anuales caracterizados por cargas externas bastante dispares (Julio de 1999-Junio de 2000 y Julio de 2000-Junio de 2001). En la Tabla 1 se muestran los modelos de masa de agua que han sido empleados en este trabajo. Para la estima de la carga máxima tolerable o carga crítica hemos utilizado las expresiones propuestas por la OCDE (1982) y Vollenweider (1976) (Tablas 1 y 2). Las variables morfométricas e hidrológicas necesarias para la aplicación de los modelos de eutrofización se han estimado de la siguiente manera: 
1) Caracteristicas morfométricas. La profundidad media $(\overline{\mathrm{z}})$, el área superficial $\left(\mathrm{A}_{\mathrm{o}}\right)$ y el volumen (V) se han calculado a partir de la medición de la altura de la lámina de agua en una regleta graduada instalada en cada laguna, así como de las correspondientes curvas hipsográficas de cada laguna (de Vicente, 1999).

2) Variables hidrológicas. El caudal de agua de entrada $\left(Q_{\text {in }}\right)$ se ha estimado considerando el caudal medio registrado en las fuentes puntuales (acequias); la precipitación directa sobre las lagunas (datos facilitados por la estación experimental "Las Palmerillas", El Ejido, Almería) y la escorrentía procedente de las ramblas. Para ello, el volumen de agua de escorrentía se cuantificó teniendo en cuenta el valor de la precipitación crítica correspondiente al tipo de litología de la cuenca vertiente ( 25 $\mathrm{mm}$ ) y el coeficiente de escorrentía (0.2) (Martín, 1997). El tiempo de retención hidráulico $\left(\mathrm{t}_{\mathrm{w}}\right)$ se calculó como la relación entre el volumen medio de la laguna y el caudal de entrada en la misma durante el período de tiempo considerado. La carga hidráulica $\left(\mathrm{q}_{\mathrm{s}}\right)$ se estimó como la relación entre la profundidad media del sistema y el tiempo de retención hidráulico. La concentración promedio anual de $\mathrm{P}\left(\mathrm{P}_{\mathrm{in}}\right)$ en el agua de entrada a las lagunas se estimó a partir de la carga externa anual de $\mathrm{P}$ y de la carga hidráulica. Por último, el coeficiente de retención (R) se estimó a partir de la carga hidráulica, siguiendo la expresión descrita por Kirchner \& Dillon (1975).

\section{Cuantificación de las tasas de sedimentación de partículas y de fósforo}

Para el análisis periódico (Julio 2000-Agosto 2001) de la cantidad y naturaleza del material sedimentado en la zona pelágica de cada laguna, se dispusieron una serie de trampas de sedimentación a 3 profundidades en el perfil vertical (a 85, 175 y $300 \mathrm{~cm}$ desde la base de las trampas). Las trampas de sedimentación consisten en cilindros dobles de metacrilato cuya relación altura/ diámetro interno $(>6)$ es suficiente para evitar la pérdida por resuspen- sión del material sedimentado (Blomqvist \& Hakanson, 1981).

El tiempo de exposición de las trampas de sedimentación fue siempre inferior a 16 días, con el fin de minimizar la mineralización del material recogido en las trampas, lo que conllevaría a una subestimación de la tasa de sedimentación de partículas.

El material sestónico, secado $\left(\mathrm{a} 60^{\circ} \mathrm{C}\right)$ y tamizado $(2 \mathrm{~mm}$ ), fue analizado para la determinación de la concentración de TP mediante la calcinación previa de la muestra y su posterior digestión ácida (Pardo et al.,1999).

\section{RESULTADOS Y DISCUSIÓN}

\section{Balance másico de fósforo}

En la figura 1 se muestra la variación temporal del volumen almacenado en ambas lagunas así como del caudal de agua de las acequias que vierten en las mismas y de la precipitación acumulada entre los sucesivos muestreos. La extraordinaria variabilidad observada en el régimen de precipitaciones, dio lugar a una continua variación en el volumen de agua de las lagunas, especialmente en el caso de la laguna Honda, para la que se ha estimado un $\mathrm{C}_{\mathrm{VAR}}$ en el volumen embalsado del $25 \%$, mientras que en la laguna Nueva el $\mathrm{C}_{\mathrm{VAR}}$ adquirió valores notablemente inferiores $(6 \%)$.

En ambas lagunas, la entrada de agua a través de las acequias de riego supuso un aporte extraordinariamente discontinuo en el tiempo.

Las lagunas de la Albufera de Adra presentaron una notable variación temporal en la masa de TP, determinada en gran medida por la considerable irregularidad observada tanto en la carga externa difusa (ramblas) como en la carga externa puntual (red de acequias) (Fig. 2). No obstante, es preciso señalar que, salvo en los momentos en los que en la laguna Honda se produjo la descarga de $\mathrm{P}$ a través de las ramblas, las variaciones en la masa de $\mathrm{P}$ en la columna de agua fueron muy superiores a las encontradas en la carga externa. Esta obser- 
vación apunta hacia el importante papel que el ciclo interno del $\mathrm{P}$ puede desempeñar en estos sistemas someros.

Como consecuencia de las fuertes lluvias características del régimen pluviométrico de la zona, la entrada de $\mathrm{P}$ procedente de las ramblas se concentró en solamente dos períodos del año (Octubre y Diciembre). Especialmente interesante resultó el incremento en la masa de TP en la laguna Honda (se multiplicó por 2.6) tras las intensas lluvias acaecidas a finales del mes de Diciembre de 2000 (57.5 mm de agua fueron registrados el día 26.12.00).

Los resultados de la aplicación del balance de masas para el $\mathrm{P}$ se presentan en la figura 3, en las que se muestra la masa de TP medida en la columna de agua en cada uno de los muestreos realizados, la masa de TP calculada (obtenida como la suma de la masa medida en el muestreo anterior y las entradas de $\mathrm{P}$ a través de las acequias y de las ramblas cuantificadas entre los sucesivos muestreos) así como la diferencia entre la masa de P medida y la calculada, lo que ha permitido evaluar la retención y la liberación de $\mathrm{P}$ desde los sedimentos.

Dada la dificultad metodológica existente para distribuir la deposición atmosférica de $\mathrm{P}$ entre los diferentes muestreos realizados, se ha optado por la no inclusión de la mísma en la representación de la figura 3 , si bien se ha tenido en cuenta para la estima anual de los procesos de retención y liberación de P (Tabla 6).

Durante el período de estudio los sedimentos de ambas lagunas actuaron como "sumidero" de P. La retención media anual de P fue de 168.5 y $29.7 \mathrm{~kg}$ en la laguna Honda y laguna Nueva, respectivamente. A partir del área superficial media de cada laguna hemos estimado una tasa media de retención de $2.10 \mathrm{~g} \mathrm{P} \mathrm{m}^{-2} \mathrm{año}^{-1}$ en la laguna Honda y de $0.11 \mathrm{~g} \mathrm{P} \mathrm{m}^{-2} \mathrm{año}^{-1}$ en la laguna Nueva.

Sin embargo, estos valores de retención son muy inferiores a la tasa de sedimentación de $\mathrm{P}$ (11.38 y $5.26 \mathrm{~g} \mathrm{P} \mathrm{m}^{-2} \mathrm{año}^{-1}$ en la laguna Honda y laguna Nueva, respectivamente) medida a partir de la instalación de las trampas de sedimentación.

En la Tabla 3 se muestran los valores de las tasas de sedimentación de TP en ambas lagunas, así como su comparación con otros ecosistemas acuáticos. Algunos autores han sugerido la existencia de una relación general entre las variables indicadoras del estado trófico, en particular la concentración de TP en la columna de agua, con el flujo de nutrientes (Tartari \& Biasci, 1997).

Las elevadas tasas de sedimentación de $\mathrm{P}$ reflejan el "stress" continuo al que se encuentran sometidas ambas lagunas, especialmente la laguna Honda. Según Margalef (1983), sedimentaciones anuales de 0.25 a 5 g P m${ }^{-2} a_{n} o^{-1}$ son características de lagos influidos por la actividad antrópica.

Llegados a este punto, es conveniente hacer notar que, como varios autores indican, la sedimentación y la retención son procesos claramente diferenciados (Fuhs, 1973; Dillon et al., 1990). La sedimentación tiene lugar en un sentido unidireccional, mediado por la fuerza de la gravedad, mientras que la retención es el resultado de procesos que operan en sentidos opuestos: la sedimentación (sentido descendente) y la liberación de $\mathrm{P}$ desde los sedimentos (sentido ascendente). Por tanto, es comprensible que la tasa de sedimentación bruta, cuantificada mediante las trampas de sedimentación, sea muy superior a la tasa de retención (sedimentación neta), obtenida a partir de la aplicación del balance de masas (Dillon et al., 1990).

En este sentido, en numerosos trabajos limnológicos, se ha reconocido la importancia de la resuspensión del sedimento no consolidado como proceso que reduce considerablemente la retención de P (Andersen \& Lastein, 1981; James \& Barko, 1997). De este modo, la resuspensión del sedimento puede provocar no sólo el transporte de P particulado desde el sedimento hacia la columna de agua sino favorecer la difusión del $\mathrm{P}$ disuelto en el agua intersticial, afectando en última instancia al estado trófico del ecosistema. Otra posible explicación a las diferenciadas generalmente observadas entre la sedimentación (bruta) y la retención de $\mathrm{P}$, sería el rápido reciclado de éste nutriente en el sedimento superficial, de modo que el $\mathrm{P}$ una vez sedimentado no es almacenado en el sedimento sino que es movilizado de nuevo hacia la 


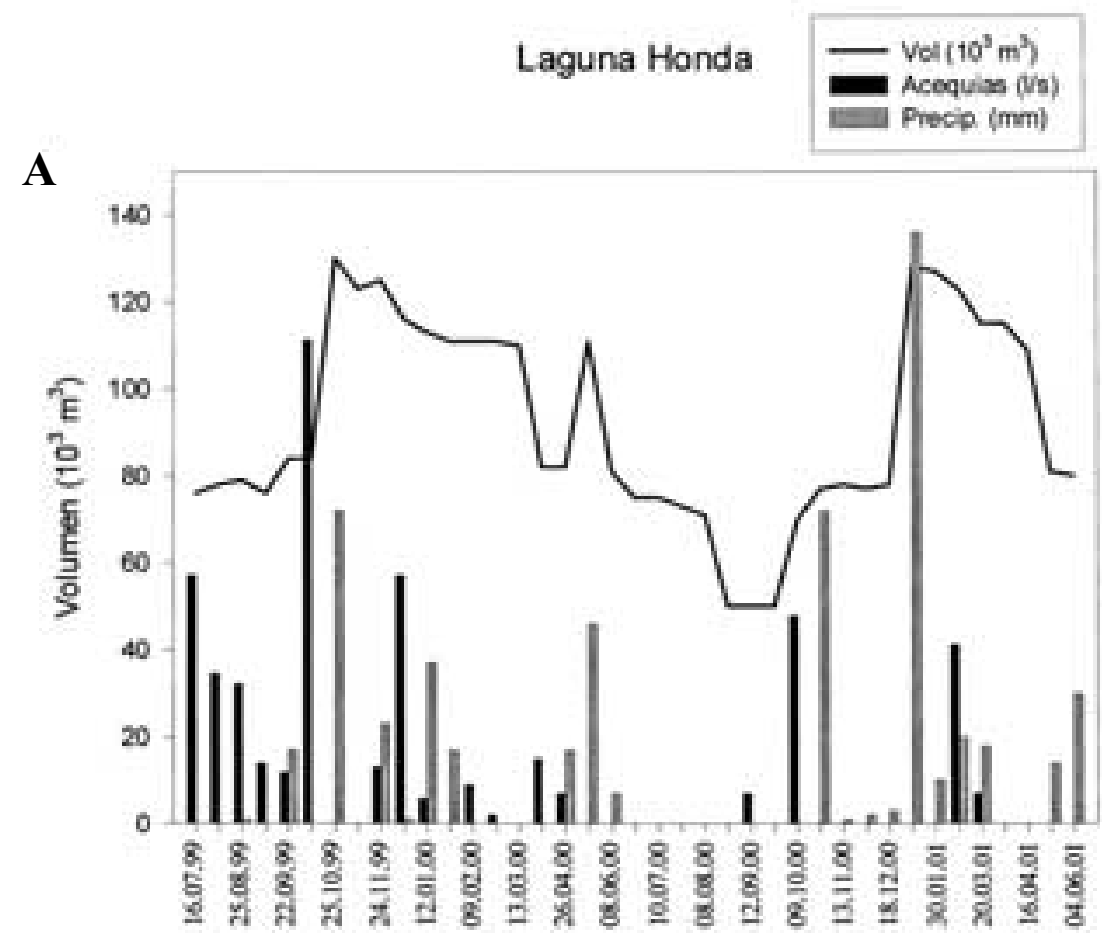

B

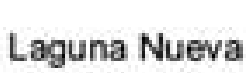

$$
\begin{aligned}
& \text { Vol }\left(10^{3} \mathrm{~m}^{3}\right) \\
& \text { Acequias (Vs) } \\
& \text { Precip. (mm) }
\end{aligned}
$$

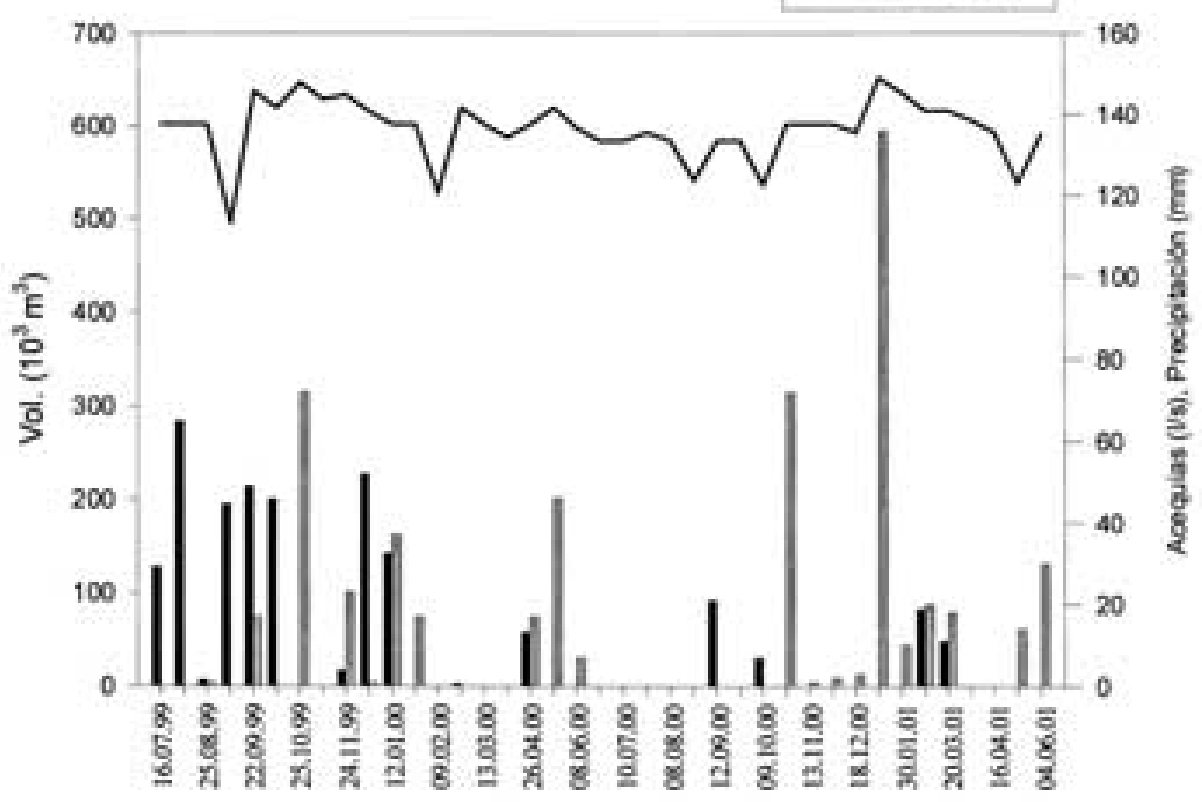

Figura 1. Variación temporal del volumen de agua de ambas lagunas, del caudal de agua vertido a través de las acequias de riego así como de las precipitación acumulada entre los muestreos sucesivos. A. laguna Honda; B. laguna Nueva. Temporal variation of the volume in both study lagoons, of the inflow from the irrigation channels and of the accumulated rainfall between consecutived samplings. A. laguna Honda; B. laguna Nueva. 
A

Laguna Honda

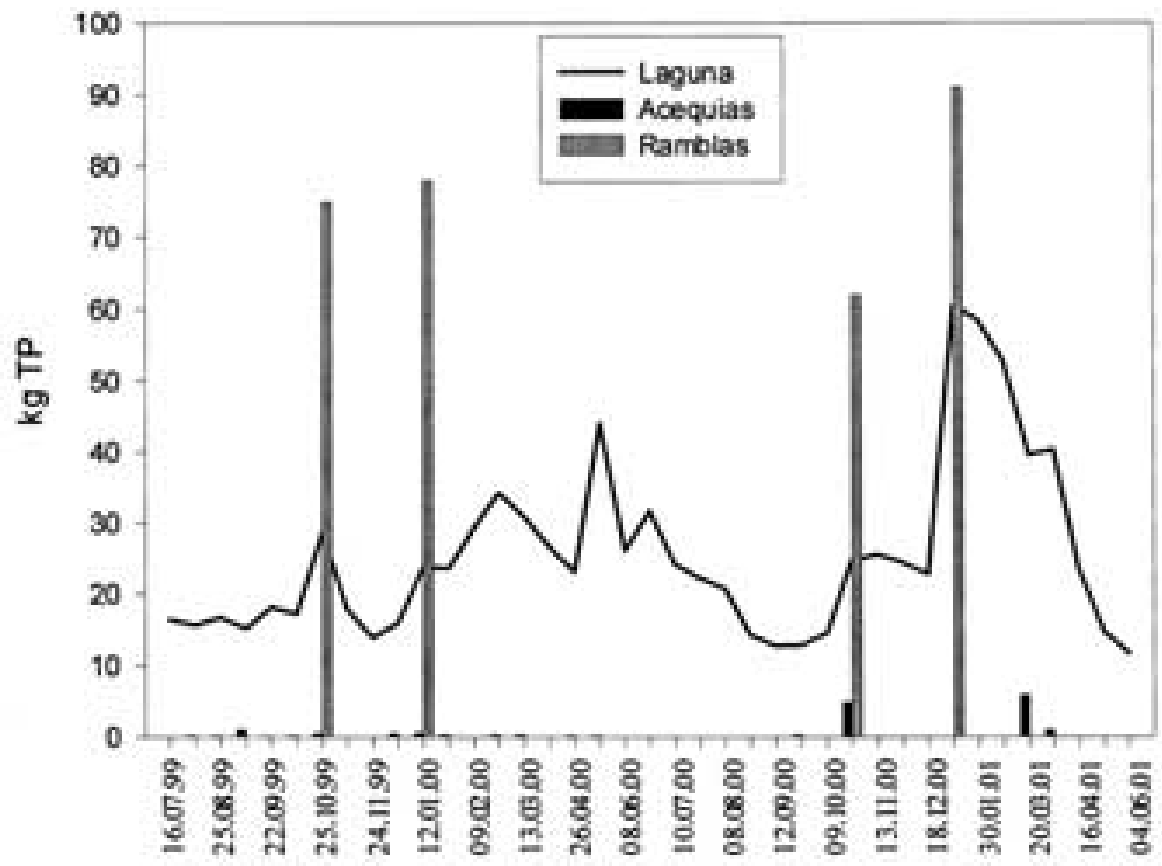

B

\section{Laguna Nueva}

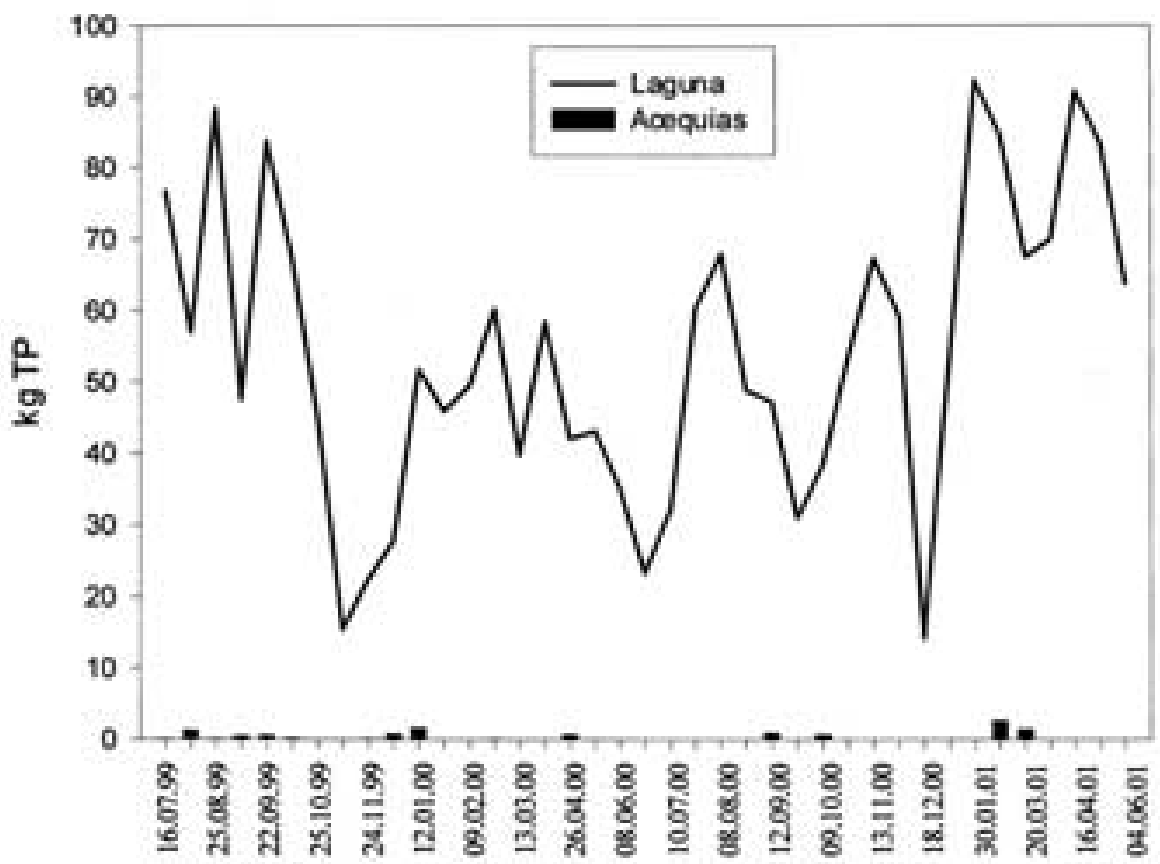

Figura 2. Evolución de la masa de TP medida en la columna de agua y de la masa de TP que entra a través de las acequias y ramblas. A. laguna Honda; B. laguna Nueva. Evolution of the TP mass measured in the water column as well as the TP mass incoming from the irrigation channels and from run off. A. laguna Honda; B. laguna Nueva. 

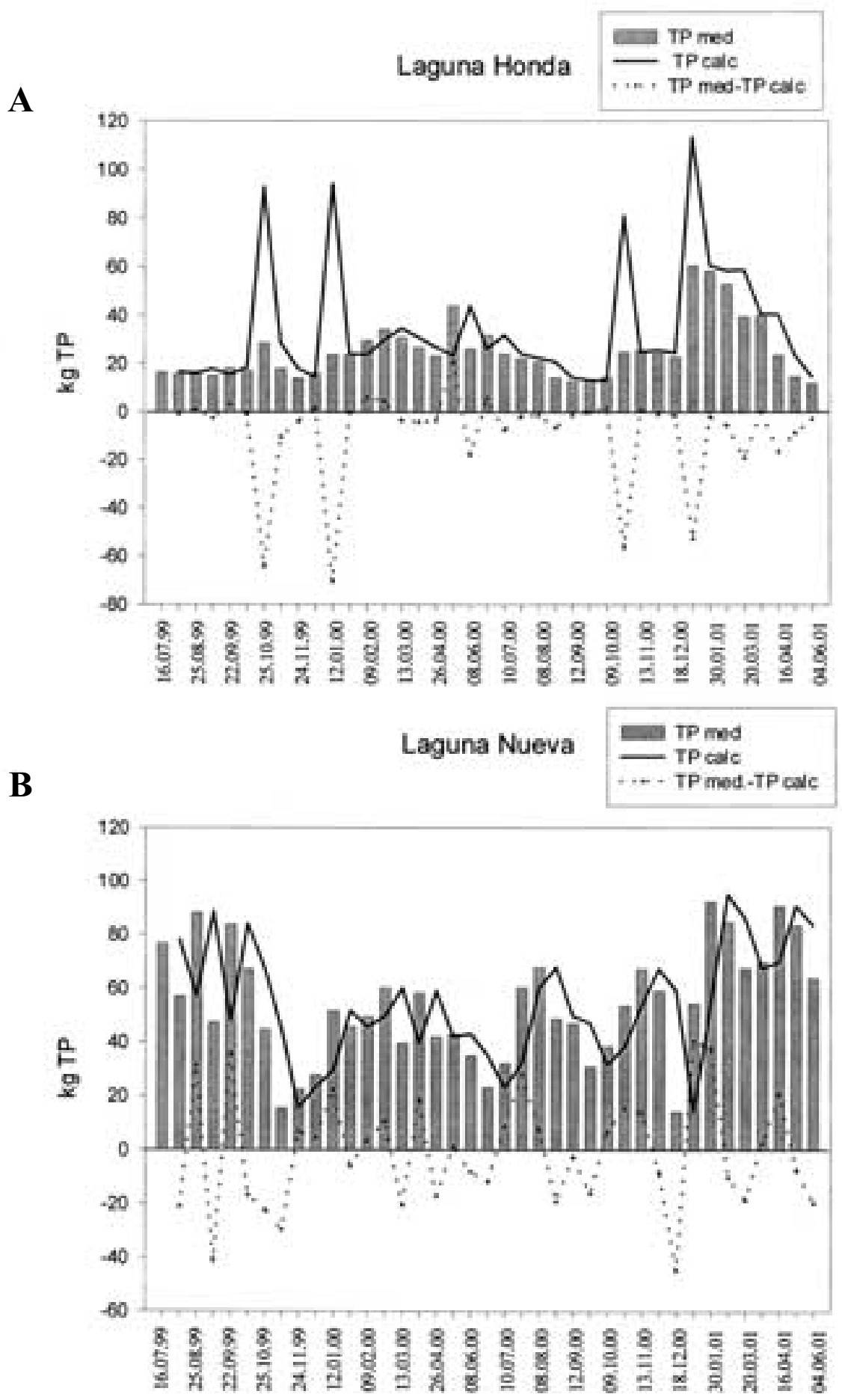

Figura 3. Resultados del balance de masas de $\mathrm{P}$ realizado en las lagunas de la Albufera de Adra. $\left(\mathrm{TP}_{\text {med }}\right.$, Masa de TP medida en la columna de agua; $\mathrm{TP}_{\mathrm{calc}}$, masa de TP calculada). A. laguna Honda; $\mathrm{B}$. laguna Nueva. Results of the TP mass balance in the lagoons from the Albufera de Adra. (TP ${ }_{\text {med }}$, measured mass of TP in the water column; TP calc, calculated mass of TP). A. laguna Honda; B. laguna Nueva. 
Tabla 3. Estudio comparativo de la tasa de sedimentación de TP en diferentes ecosistemas acuáticos. (O: oligotrófico; O-M: oligotróficomesotrófico; M: mesotrófico; E: eutrófico; E-H: eutrófico-hipereutrófico; HE: hipereutrófico). Comparative study of TP sedimentation fluxes. (O: oligotrophic; O-M:oligotrophic-mesotrophic; M: mesotrophic; E: eutrophic; E-H: eutrophic-hipereutrophic; HE: hipereutrophic).

\begin{tabular}{|c|c|c|c|}
\hline Lago & Estado trófico & $\begin{array}{c}\text { TP } \\
(\mu \mathrm{g} / \mathrm{l})\end{array}$ & $\begin{array}{l}\text { Tasa de sed. de TP } \\
\quad\left(\mathrm{mg} \mathrm{m}^{-2} \text { día }^{-1}\right)\end{array}$ \\
\hline Fayettville Green (USA) ${ }^{1}$ & $\mathrm{O}$ & 0.4 & 0.4 \\
\hline Orta (Italia) ${ }^{1}$ & $\mathrm{O}-\mathrm{M}$ & 5 & 5.1 \\
\hline Chuzenji (Japón) ${ }^{1}$ & $\mathrm{O}$ & 6 & 3.2 \\
\hline Erie (USA-Canadá) ${ }^{1}$ & $\mathrm{M}$ & 16 & 3.4 \\
\hline Zurich (Suiza) ${ }^{1}$ & M & 65 & 5.4 \\
\hline Norvikken (Suecia) ${ }^{1}$ & $\mathrm{E}$ & 66 & 19.7 \\
\hline Sempach $(\text { Suiza })^{2}$ & E & 90 & 5.8 \\
\hline Nueva (España) ${ }^{3}$ & E & 94 & 14.4 \\
\hline Onondaga (USA) ${ }^{1}$ & $\mathrm{HE}$ & 100 & 29.4 \\
\hline Lugano (Suiza) ${ }^{1}$ & E-H & 132 & 2.8 \\
\hline Zug (cubeta Sur, Suiza) ${ }^{1}$ & - & 205 & 6.1 \\
\hline Honda $(\text { España })^{3}$ & $\mathrm{HE}$ & 262 & 31.2 \\
\hline Rotsee (Suiza) ${ }^{1}$ & - & 369 & 5.9 \\
\hline
\end{tabular}

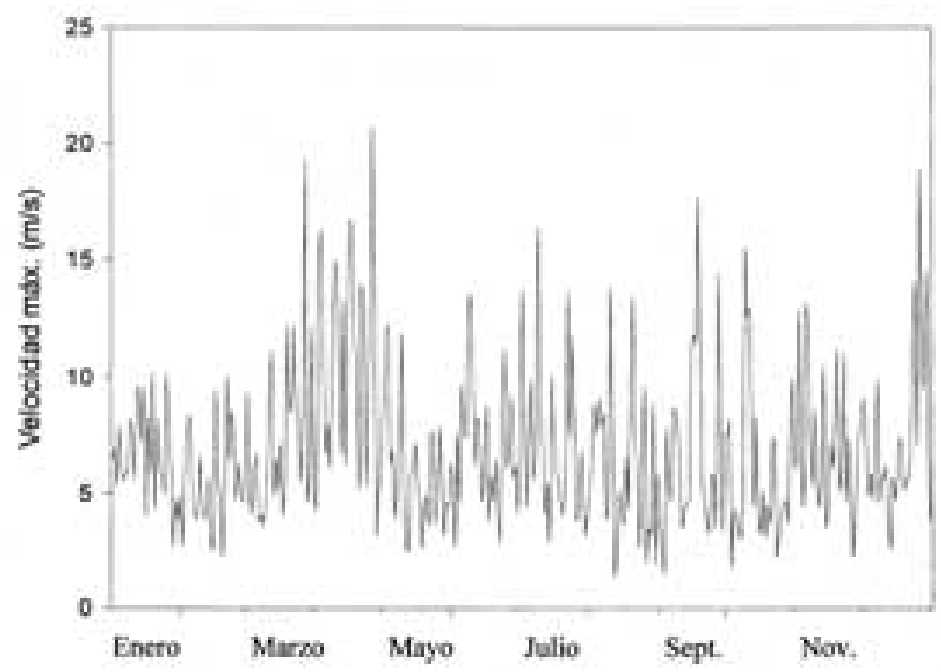

Figura 4. Velocidad máxima diaria del viento (Enero-Diciembre 2000). Maximum wind speed (January-December 2000).

columna de agua (Dillon et al., 1990). El análisis de la situación a pequeña escala (periodicidad aproximadamente quincenal) reveló la extraordinaria variabilidad temporal en los procesos de retención y liberación de $\mathrm{P}$, propios de sistemas áltamente fluctuantes como son las lagunas de la Albufera de Adra.

En la laguna Honda se puede apreciar que la mayor retención de $\mathrm{P}$ coincidió con la entrada espectacularmente importante de $\mathrm{P}$ a través de las ramblas (meses de Octubre y Diciembre de los años 1999 y 2000). Por el contrario, la máxima liberación de $\mathrm{P}$ en esta laguna se detectó durante la primavera de 2000 (47\% de la masa anual de $\mathrm{P}$ liberada). El agente responsable del patrón observado fue el fuerte viento registrado a finales del mes de Abril (superior a $\operatorname{los} 20 \mathrm{~m} / \mathrm{s}$ ) (Fig. 4), que causó la resuspensión del sedimento superficial de la laguna Honda, incrementándose en un $90 \%$ la masa de TP medida en la columna de agua. Sin embargo, en la laguna Nueva no se apreció un 


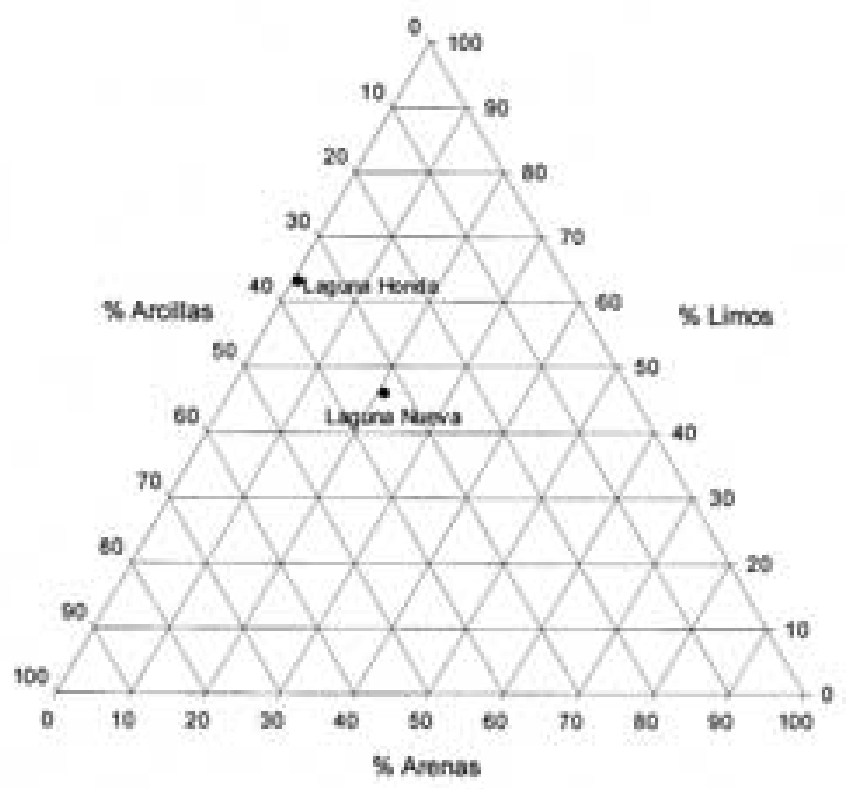

Figura 5. Composición granulométrica del sedimento superficial de la laguna Honda y de la laguna Nueva (Arcillas: < $2 \mu$ m; Limos: 2-50 $\mu \mathrm{m}$ y Arenas: $>50 \mu \mathrm{m})$. Granulometric composition of the surficial sediment of both study lagoons (Clay: < $2 \mu \mathrm{m} ;$ Silt: 2-50 $\mu \mathrm{m}$ and Sand: $>50 \mu \mathrm{m})$.

incremento significativo en la masa de TP en la columna de agua durante ese periodo, resultado probablemente de la diferente composición granulométrica del sedimento superficial de ambas lagunas (Fig. 5).

En lo que se refiere a la laguna Nueva es difícil encontrar un patrón claro de comportamiento de los procesos de retención y de liberación de P. Resulta especialmente interesante la elevada tasa media anual de liberación de TP desde el sedimento de la laguna Nueva (316.1 kg TP frente a los $44.4 \mathrm{~kg}$ TP liberados anualmente por el sedimento de la laguna Honda). Como es bien sabido, el término "carga interna" incluye no sólo al intercambio de $\mathrm{P}$ entre el sedimento superficial, de la zona pelágica y litoral, y la columna de agua, sino que engloba tambien a flujos más profundos, subterráneos. En este sentido es necesario comentar que los trabajos realizados sobre el régimen hidrológico de las lagunas de la Albufera de Adra han señalado la importancia de la descarga de agua subterránea, procedente del acuífero del Delta del Río Adra, hacia la laguna Nueva (Benavente, 2002), flujo que en última instancia puede haber contribuido a las elevadas tasas de liberación de P estimadas desde el sedimento de esta laguna. Sin embargo, cuando se han cuantificado los flujos de $\mathrm{P}$ a nivel de la interfase agua-sedimento, mediante la realización de experiencias de laboratorio, se han observado máximas tasas de liberación de $\mathrm{P}$ desde el sedimento superficial de la laguna Honda (de Vicente et al., 2001).

En la laguna Nueva fue posible reconocer procesos especialmente revelantes para la dinámica del P, como el de la precipitación biogénica de calcita. Este fenómeno fue detectado, en un primer momento, por la recogida de cristales de $\mathrm{CaCO}_{3}$ en las trampas de sedimentación localizadas en el estrato más superficial de la columna de agua, a finales del mes de Agosto de 2000 (de Vicente, 2001). Como era esperable, el índice de saturación en calcita reflejó condiciones de sobresaturación en las capas superficiales $(10 \mathrm{~cm})$ durante el mes de Julio y Agosto de 2000 (Tablas 4 y 5). El proceso de precipitación biogénica de calcita se ve favorecido durante la época estival, bajo condiciones de valores elevados de $\mathrm{pH}$ y temperatura. Precisamente durante el mes de Agosto de 2000, 
Tabla 4. Índice de saturación $(\log \mathrm{AP} / \mathrm{K})$ de diferentes minerales en la laguna Nueva durante el verano de 2000. Saturation index of different minerals in laguna Nueva during the summer 2000.

\begin{tabular}{lcccccc}
\hline & Anhidrita & Calcita & Aragonito & Dolomita & Magnesita & Yeso \\
\hline Junio & -0.839 & - & - & - & - & -0.625 \\
Julio & -0.756 & +1.154 & +1.013 & +2.445 & +0.835 & -0.552 \\
Agosto & -0.764 & +1.208 & +1.066 & +2.579 & +0.894 & - \\
Septiembre & -0.727 & - & - & - & -0.514 \\
\hline
\end{tabular}

en la laguna Nueva se alcanzaron temperaturas superiores a $\operatorname{los} 28^{\circ} \mathrm{C}$ y valores de $\mathrm{pH}$ de hasta 8.5 en el estrato más superficial de la columna de agua. Como muchos autores han sugerido (Wetzel, 1975; Koschel et al., 1983 Kleiner, 1990) el P puede ser retirado de la columna de agua mediante su adsorción sobre los cristales de $\mathrm{CaCO}_{3}$. De hecho, la máxima tasa de sedi-

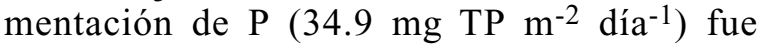
medida en la trampa de sedimentación situada en la capa más superficial durante el mes de Agosto de 2000. La espectacular sedimentación de $\mathrm{P}$ detectada durante ese mes supuso una reducción del $28 \%$ en la masa de TP de la columna de agua (Fig. 3).

Durante el período de estudio se han observado acusados incrementos y descensos en la masa de TP de la columna de agua. En este sentido es interesante resaltar la enorme reducción (76\%) en la masa de TP de la laguna Nueva entre finales de Noviembre y principios de Diciembre de 2000. Los análisis para la determinación de la concentración de TP en el sedimento superficial confirmaron la extraordinaria retención de $\mathrm{P}$ por el sedimento durante ese período (de Vicente et al.,2002). Por último, es interesante mencionar el notable incremento observado en la masa de TP de la laguna Nueva tras las lluvias acaecidas a finales del mes de Diciembre de 2000. Aunque esta laguna no recibe la descarga directa del agua de escorrentía (ramblas), durante esas lluvias se alcanzaron niveles extremadamente elevados en la laguna Honda que desencadenaron la descarga puntual de agua desde dicha laguna hacia la laguna Nueva, a través de una de las acequias de riego (Paracuellos, com.pers.).

\section{Aplicación de Modelos de Eutrofización y de Carga crítica}

En la Tabla 6 se observa la participación de cada una de las fuentes externas en la carga externa anual de P. En la laguna Honda la carga externa difusa representó el $93.8 \%$ y el $89.9 \%$ de la carga externa anual de $\mathrm{P}$ en los períodos Julio 1999- Junio 2000 y Julio 2000- Junio 2001, respectivamente. Entre el periodo 19992000 y el periodo 2000-2001, se pudo apreciar un ligero aumento en la carga externa de P que llegó a la laguna Honda, asociado directamente a un incremento en la entrada de $\mathrm{P}$ a través de la red de acequias.

La laguna Nueva, que no recibe la descarga procedente de ninguna rambla, se vió afectada en mayor medida por la variación interanual en el caudal de las fuentes puntuales (acequias). De

Tabla 5. Variación vertical del I. S. en la laguna Nueva durante el mes de Agosto 2000. Vertical variation of the saturation index in laguna Nueva during August 2000.

\begin{tabular}{lcccccc}
\hline & Anhidrita & Calcita & Aragonito & Dolomita & Magnesita & Yeso \\
\hline $\mathbf{1 0} \mathbf{~ c m}$ & -0.764 & +1.208 & +1.066 & +2.579 & +0.894 \\
$\mathbf{1 5 0} \mathbf{~ c m}$ & -0.826 & - & - & - & - & -0.622 \\
$\mathbf{3 0 0} \mathbf{~ c m}$ & -1.032 & - & - & - & -0.818 \\
\hline
\end{tabular}


Tabla 6. Resultados de la estima de la carga externa de fósforo a las lagunas a partir de distintas fuentes. Quantification of the different $P$ external loads to the studied systems.

\begin{tabular}{lcccc}
\hline & \multicolumn{2}{c}{ Laguna Honda } & \multicolumn{2}{c}{ Laguna Nueva } \\
& $\mathbf{1 9 9 9 - 2 0 0 0}$ & $\mathbf{2 0 0 0 - 2 0 0 1}$ & $\mathbf{1 9 9 9 - 2 0 0 0}$ & $\mathbf{2 0 0 0 - 2 0 0 1}$ \\
\hline Deposición atmosférica (kg P) & 5.4 & 5.1 & 17.3 & 17.1 \\
Carga externa puntual (kg P) & 4.6 & 12.1 & 6.3 & 5.9 \\
Carga externa difusa (kg P) & 152.6 & 152.6 & 0 & 0 \\
Carga externa (kg P) & 162.6 & 169.8 & 23.6 & 23.0 \\
Carga externa (mg P $^{-2} \mathbf{a n ̃ o}^{-\mathbf{1}}$ ) & 1968.9 & 2188.1 & 90.2 & 88.7 \\
\hline
\end{tabular}

este modo, la carga externa puntual de $\mathrm{P}$ a esta laguna se redujo ligeramente $(6 \%)$ entre el periodo 1999-2000 y 2000-2001.

En la Tabla 7 se muestran los valores de las variables morfométricas (profundidad media, área superficial y volumen medio) e hidrológicas (carga hidráulica, tiempo de retención, caudal de entrada y coeficiente de retención), así como la carga externa y la concentración media de $\mathrm{P}$ en el agua de entrada a las lagunas durante los períodos de estudio considerados. Como puede observarse, los caudales de entrada a ambas lagunas se redujeron notablemente entre el periodo 19992000 y el $2000-2001$ (en un $13 \%$ y $45 \%$ en la laguna Honda y laguna Nueva, respectivamente). Esta modificación en el caudal de entrada a las lagunas supuso una reducción en la carga hidráulica así como un incremento en el tiempo y en el coeficiente de retención.

A pesar de la reducción en el caudal de entrada, la carga externa de $\mathrm{P}$ a la laguna Honda se mantuvo en niveles muy elevados durante el periodo 2000-2001, como consecuencia de la alta concentración de $\mathrm{P}$ en el agua de entrada. Es interesante destacar el hecho de que el acusado descenso experimentado en el caudal de agua de entrada a la laguna Nueva no se reflejó en una modificación del volumen de agua de la laguna, una situación que podría explicarse por la ya comentada relevancia de la descarga de agua subterránea en esta laguna.

En la Tabla 8 se observan los resultados de la aplicación de los modelos de masa de agua ensayados. Como se puede apreciar, los modelos de masa de agua más comúnmente empleados no presentaron un ajuste deseable y no permitieron predecir la concentración promedio anual de fósforo medida en las lagunas. En la laguna Honda, la concentración predicha fue, según el modelo, entre un 39 y un $75 \%$ la concentración real de TP. De igual modo, los valores predichos para la laguna Nueva oscilaron

Tabla 7. Valores de las variables más importantes incluidas en los modelos de masa de agua. Variables used in the empirical eutrophication models.

\begin{tabular}{|c|c|c|c|c|}
\hline & \multicolumn{2}{|c|}{ Laguna Honda } & \multicolumn{2}{|c|}{ Laguna Nueva } \\
\hline & 1999-2000 & 2000-2001 & 1999-2000 & 2000-2001 \\
\hline$\overline{\mathbf{z}}(\mathbf{m})$ & 1.17 & 1.11 & 2.29 & 2.28 \\
\hline$A_{0}\left(m^{2} 10^{3}\right)$ & 82.6 & 77.6 & 261.5 & 259.3 \\
\hline$V\left(m^{3} 1^{3}\right)$ & 96.6 & 86.5 & 598.4 & 590.9 \\
\hline$Q_{\text {in }}\left(m^{3} / \mathbf{a n ̃ o} 10^{3}\right)$ & 674.1 & 584.9 & 560.5 & 306.1 \\
\hline $\mathbf{L}_{e x t}\left(\mathrm{mg} \mathrm{m}^{-2} \mathbf{a} \tilde{n} 0^{-1}\right)$ & 1968.9 & 2188.1 & 90.2 & 88.7 \\
\hline$P_{\text {in }}(\mu \mathrm{g} / \mathrm{l})$ & 235.5 & 295.7 & 42.2 & 75.5 \\
\hline 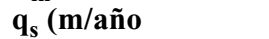 & 8.36 & 7.40 & 2.14 & 1.18 \\
\hline$t_{w}(a \tilde{o n s})$ & 0.14 & 0.15 & 1.07 & 1.93 \\
\hline $\mathbf{R}$ & 0.57 & 0.59 & 0.80 & 0.88 \\
\hline
\end{tabular}


Tabla 8. Resultados de la aplicación de los modelos de masa de agua. ( $\mu \mathrm{g} \mathrm{P} / 1)$. Results of the empirical models validation $(\mu g / l)$.

\begin{tabular}{lcccr}
\hline & \multicolumn{2}{c}{ Laguna Honda } & \multicolumn{2}{c}{ Laguna Nueva } \\
& $\mathbf{1 9 9 9 - 2 0 0 0}$ & $\mathbf{2 0 0 0 - 2 0 0 1}$ & $\mathbf{1 9 9 9 - 2 0 0 0}$ & $\mathbf{2 0 0 0 - 2 0 0 1}$ \\
\hline Dillon \& Rigler (1974) & 101 & 121 & 8 & 9 \\
Vollenweider (1976) & 172 & 213 & 21 & 32 \\
OCDE (1982) & 105 & 126 & 19 & 26 \\
Foy (1992) & 184 & 228 & 21 & 31 \\
Concentración promedio & 244 & 304 & 84 & 100 \\
anual de TP medida & & & & \\
\hline
\end{tabular}

entre un 9 y un $32 \%$ de la concentración de TP medida en la laguna. De entre todos los modelos ensayados es el modelo propuesto por Foy (1992) el que proporcionó los mejores ajustes en ambas lagunas. El desajuste observado entre la concentración real de TP y la predicha por los modelos de eutrofización puede ser el resultado de la no inclusión de la carga interna en los mismos. Aunque, como indican los resultados del balance de masas de $\mathrm{P}$, sobre una base anual ambas lagunas retienen $\mathrm{P}$, existen episodios periódicos en los que la liberación de $\mathrm{P}$ alcanza valores significativos. En este sentido, Osgood (1988) señaló que en lagos polimícticos, donde la razón $\mathrm{z} /\left(\mathrm{A}_{\mathrm{o}}\right)^{1 / 2}$ era inferior a 3 , la concentración promedio estival de TP medida en superficie se ajustaba considerablemente bien a la predicha por aquellos modelos que incluyen tanto a la carga externa como a la carga interna de P. De igual modo, Nürnberg (1984) sugirió la inclusión de la carga interna en los modelos que tratan de predecir la concentración promedio anual de TP en la masa de agua. Si bien es cierto que este tipo "general" de modelos no proporciona ajustes "perfectos" a situaciones particulares, el pronóstico se ve debilitado en nuestro caso particular en que es difícilmente comprobable la primera de las asunciones de partida requeridas para su aplicación (el mantenimiento de un estado estacionario) (Harper, 1992), al no existir salidas superficiales.

En todo caso, estos resultados sugieren y ponen el acento en la necesidad de: a) Cuantificar otras fuentes de entrada (externas e internas) de fósforo al sistema que pueden llegar a jugar un papel esencial. Entre éstas podemos encontrar las provenientes de las deyecciones de vertebrados acuáticos (en particular, aves), las que pueden asociarse al funcionamiento de la zona litoral o la liberación de $\mathrm{P}$ desde el sedimento (Nürnberg, 1998).

b) Comprobar la fiabilidad de las estimas de carga de Fósforo al sistema a través de fuentes difusas como las que suponen la escorrentía superficial que alcanza las lagunas (en este caso, a la laguna Honda) a través de las ramblas pues aunque en forma de pulsos irregulares e impredecibles, representan un porcentaje muy elevado de la carga anual que aquellas reciben. En este sentido, la calibración de los coeficientes "genéricos" de exportación de nutrientes propuestos por la bibliografía, a la realidad de la cuenca de captación de las lagunas, se presenta como una necesidad perentoria.

c) Explorar y considerar (cuantificar) las relaciones entre el agua de las lagunas y el agua subterránea ( $\mathrm{y}$, a través de ésta, entre las lagunas) como términos que "afinen" los valores de las cargas (hidráulica y de nutrientes) de entrada y salida a cada uno de los sistemas, especialmente en el caso de la laguna Nueva.

Por último, el conocimiento de la carga crítica es una herramienta esencial para la gestión de los ecosistemas acuáticos, sobre todo si el objeti- 
Tabla 9. Comparación entre la carga crítica de $\mathrm{P}$ predicha por los modelos y la carga externa anual de $\mathrm{P}$ a los sistemas ( $\mathrm{mg} \mathrm{m}^{-2}$ año $\left.{ }^{-1}\right)$. Comparison of the predicted critical $P$ loading values and the measured annual external P load $\left(\mathrm{mg} \mathrm{m}^{-2}\right.$ year $\left.{ }^{-1}\right)$.

\begin{tabular}{lcccc}
\hline & \multicolumn{2}{c}{ Laguna Honda } & \multicolumn{2}{c}{ Laguna Nueva } \\
& $\mathbf{1 9 9 9 - 2 0 0 0}$ & $\mathbf{2 0 0 0 - 2 0 0 1}$ & $\mathbf{1 9 9 9 - 2 0 0 0}$ & $\mathbf{2 0 0 0 - 2 0 0 1}$ \\
\hline Carga externa & 1968.9 & 2188.1 & 90.2 & 88.7 \\
OCDE (1982) & 229.8 & 205.3 & 87.1 & 94.2 \\
Vollenweider (1976) & 64.7 & 58.9 & 37.4 & 26.1 \\
\hline
\end{tabular}

vo último consiste en reducir el estado trófico de la masa de agua a través del control de la carga externa.En este sentido, es bien sabido que una misma carga externa de P puede resultar permisible o excesiva según las características morfométricas (profundidad media, carga hidráulica y tiempo de renovación) del sistema receptor. Si la carga externa es superior a la carga crítica, el sistema estará acumulando $\mathrm{P}$ y eventualmente aumentando su nivel trófico, mientras que si es inferior, el estado trófico del sistema no cambiará y, a largo plazo, podría reducirse.

Los resultados de la aplicación de los modelos de carga crítica han revelado que la laguna Honda recibió una carga de fósforo de 9 a 37 veces superior a la considerada tolerable (crítica) (Tabla 9) y, por ello, una reducción de esta carga externa se destaca como el primer paso a seguir en cualquier estrategia de gestión que pretenda ir más allá de ser una medida cosmética (de minimización de efectos antes que de reducción de las causas). Sin embargo, la laguna Nueva se encontró sometida a cargas externas muy inferiores y, dependiendo del modelo que se considere, los valores de la carga externa excedieron o no la carga permisible a esta laguna.

A partir de los resultados que hemos obtenido, concluimos que:

1. Aunque sobre una base anual las lagunas de la Albufera de Adra retienen P en el sedimento, es posible identificar períodos especialmente relevantes de liberación de éste nutriente desde el sedimento de ambas lagunas.

2. La irregularidad en el régimen pluviométrico, la frecuencia de fuertes vientos en la zona y el carácter somero de las lagunas podrían explicar la extraordinaria variabilidad temporal observada en la masa de TP en la columna de agua.

3. Las acusadas diferencias reconocidas entre las tasas de sedimentación y las de retención de $\mathrm{P}$ en ambas lagunas han puesto de manifiesto la relevancia de la regeneración bentónica de nutrientes en estas lagunas.

4. El desajuste existente entre la concentración de TP medida en la columna de agua y la predicha por los modelos de masa de agua puede ser resultado de la no inclusión de la carga interna de $\mathrm{P}$ en los mismos.

5. Por último, el estado trófico de las lagunas (hiper/eutrófico) es consecuencia de la excesiva carga externa anual de $\mathrm{P}$ que están recibiendo las lagunas, especialmente la laguna Honda. Así mismo, la recepción de una mayor carga externa de $\mathrm{P}$ junto con la menor profundidad media de esta laguna la hacen especialmente vulnerable frente a un continuo proceso de eutrofización.

\section{AGRADECIMIENTOS}

Este trabajo ha sido financiado por la Consejería de Medio Ambiente de la Junta de Andalucía (Proyecto UE-LIFE B4-3200/98/458 "Conservación de las Albuferas de Adra") y por el proyecto CICYT HID 99-0836.

\section{BIBLIOGRAFÍA}

AHLGREN, I., T. FRISK \& L. KAMP-NIELSEN. 1988. Empirical and theorical models of phospho- 
rus loading, retention and concentration vs. lake trophic state. Hydrobiologia, 170: 285-303.

ANDERSEN, F. Ø. \& E. LASTEIN. 1981. Sedimentation and resuspension in shallow eutrophic Lake ArreskØv, Denmark. Verh. Internat. Verein. Limnol., 21: 425-430.

BENAVENTE, J. 2002. Estudio hidrológico de las Albuferas de Adra (Almería) y su entorno. In: Agricultura y Medio Ambiente en el entorno de las Albuferas de Adra. J. C. Nevado \& M. Paracuellos (eds): 67-75. Consejería de Medio Ambiente, Almería. España.

BLOMQVIST, S. \& L. HAKANSON.1981. A review on sediment traps in aquatic environments. Arch. Hydrobiologia, 91:101-132.

CALZADA-BUJAK, I., L. SERRANO, J. TOJA \& T. L. CRISMAN. 2001. Phosphorus dynamics in a Mediterranean temporary pond, Doñana National Park, Spain. Verh. Internat. Verein. Limnol., 27: 3986-3991.

CRUZ-PIZARRO, L., M. ARGAIZ, I. GARZÓN \& J. LÓPEZ. 1992. Batimetría de las lagunas de la Albufera de Adra. Informe técnico. Instituto del Agua-Universidad de Granada.

CRUZ-PIZARRO, L., I. DE VICENTE, E. MORENO-OSTOS, V. AMORES \& K. EL MABROUKI. 2003. Estudios de diagnóstico y viabilidad en el control de la eutrofización de las lagunas de la Albufera de Adra. Limnetica, 23: 137-156.

DE VICENTE, I. 1999. Control de la eutrofización en las Albuferas de Adra (Almería). Papel del sedimento en el ciclo del fósforo. Proyecto Fin de Carrera, Universidad de Granada. 73 pp.

DE VICENTE, I. 2001. Regeneración bentónica de nutrientes ( $\mathrm{C}, \mathrm{N}$ y $\mathrm{P}$ ) en las Albuferas de Adra (Almería). Proyecto de Investigación, Universidad de Granada. 74 pp.

DE VICENTE, I., V. AMORES, K. EL MABROUKI, E. MORENO, I. RODRÍGUEZ-PARÍS \& L. CRUZ-PIZARRO. 2001. Balance de fósforo en las lagunas de las Albuferas de Adra (Almería, España). Actas del V Simposio sobre el Agua en Andalucia, 3: 301-311.

DE VICENTE, I., L. SERRANO, V. AMORES, V. CLAVERO \& L. CRUZ-PIZARRO. 2001. Sediment phosphate fractionation and interstitial water phosphate concentrations in two coastal lagoons (Albuferas de Adra, SE Spain). Hydrobiologia, 492: 95-105.

DILLON, P. J. \& F. H. RIGLER. 1974. A test of a simple nutrient budget model predicting the phos- phorus concentration in lake water. J. Fish. Res. Bd. Can., 31:1771-1778.

DILLON, P. J., R. D. EVANS \& L. S. MOLOT. 1990. Retention and resuspension of phosphorus, nitrogen and iron in a Central Ontario lake. Can. J. Fish. Aquat. Sci., 47: 1269-1274.

FOY, R. H. 1992. A phosphorus loading model for Northern Irish lakes. Wat. Res., 26: 633-638.

FUHS, G. W. 1973. Improved device for the collection of sedimenting matter. Limnol. Oceanogr., 18: 989-993.

GARCÍA-RUIZ, R., G . PARRA, F. GUERRERO \& J. LUCENA. 2001. Sedimentation and phosphorus fractions and temporal variation in the $\mathrm{C}: \mathrm{P}$ ratio in La Concepción reservoir, southern Spain. New Zealand Journal of Marine and Freshwater Research, 35: 711-723.

GOLTERMAN, H. 1995. The labyrinth of nutrient cycles and buffers in wetlands. Results based on research in the Camargue (southern France). Hydrobiologia, 315:39-58.

HARPER, D. 1992. Eutrophication of freshwaters. Principles, problems and restoration. London: Chapman \& Hall.

HENDRY, C. D., P. L. BREZONIK \& E. S. EDGERTON. 1981. Atmospheric deposition of nitrogen and phosphorus in Florida. In: Atmospheric Pollutans in Naturals Waters. S. J. Eisenreich (ed.):199-215. Ann Arbor Science Publishers, Ann Arbor, Michigan, USA.

HUPFER, M., R. GÄCHTER \& R. GIOVANOLI. 1995. Transformations of phosphorus species in settling seston and during early diagenesis. Aquatic Sciences, 57: 305-323.

JAMES, W. F. \& J. W. BARKO. 1997. Net and gross sedimentation in relation to the phosphorus budget of Eau Galle Reservoir, Wisconsin. Hydrobiologia, 345: 15-20.

KALFF, J. 2002. Limnology. New Jersey: Prentice Hall.

KLEINER, J. 1990. Calcite precipitation- regulating mechanism in hardwater lakes. Verh. Internat. Verein. Limnol., 24: 136-139.

KIRCHNER, W. B. \& P. J. DILLON. 1975. An empirical method for estimating the retention of phosphorus in lakes. Water Resour. Res., 11: 182-83.

KOSCHEL, R., B. JURGEN, G. PROFT \& F. RECKNAGEL. 1983. Calcite precipitation as a natural control mechanism of eutrophication. Arch. Hydrobiol., 98: 380-408.

LAMPERT, W. \& U. SOMMER. 1997. Limnoecology: the ecology of lakes and streams. Oxford University Press. U. K. 
MARGALEF, R. 1983. Limnología. Ed. Omega. Barcelona.

MARTÍN ROSALES, W. 1997. Efectos de los diques de retención en el borde meridional de la Sierra de Gador (Almería). Tesis Doctoral, Universidad de Granada. 266 pp.

MURPHY, J \& J. P. RILEY. 1962. A modified single solution method for the determination of posphate in natural waters. Anal. Chim. Acta, 27: 31-36.

NÜRNBERG, G. K. \& R. H. PETERS. 1984. The importance of the internal phosphorus load to the eutrophication of lakes with anoxic hypolimnia. Ver. Internat. Verein. Limnol. 22:190-194.

NÜRNBERG, G. K. 1998. Prediction of annual and seasonal phosphorus concentrations in stratified and polymictic lakes. Limnol. Oceanogr., 43 (7):1544-1552.

OECD (Organization for Economic Coopertion and Development). 1982. Eutrophication of Waters. Monitoring, Assesment and Control. Final report. OECD Cooperative Programme on Monitoring of Inland Waters ( Eutrophication Control), Environment Directorate, OECD, París. 154 p.

OGLESBY, R. T. \& W. R. SCHAFFNER. 1978. Phosphorus loadings to lakes and some of their responses. Part 2. Regression models of summer phytoplankton standings crops, winter total $\mathrm{P}$ and transparency of New York lakes with known phosphorus loadings. Limnol. Oceanogr., 23: 135-45.

OSGOOD, R. A. 1988. Lake mixis and internal phosphorus dynamics. Arch. Hydrobiol., 113: 629-638.

PARDO, P., J. F. LÓPEZ-SÁNCHEZ, G. RAURET, V. RUBAN, H. MUNTAN \& PH. QUEVAUILLER. 1999. Study of the stability of extractable phosphate content in a candidate reference material using a modified Williams extraction procedure. The Analyst, 124: 407-411.

RAST, W. \& G. F. LEE. 1978. Summary analysis of the North American (US portion) OECD Eutrophication Project: Nutrient loading-lake res- ponse relationships and trophic state indices. Ecological Research Series, No. EPA-600/3-78008,US Environmental Protection Agency, Environmental Research Laboratory, Corvallis, Oregon, USA.

REYNOLDS, C. S. 1984. The ecology of freshwater phytoplankton. Cambridge University Press. U. K.

ROBINSON, G. W. 1922. New method for mechanical analysis of soil and another dispersion. J. Agr. Ac., 12: 306-321.

RYDING, S. O. \& W. RAST. 1992. El control de la eutrofización en lagos y pantanos. Madrid: Pirámide.

SAKAMOTO, M. 1966. Primary production by the phytoplankton community in some Japanese lakes and its dependence on lake depth. Arch. Hydrobiol., 62: 1-28.

SØNDERGAARD, M. 1989. Phosphorus release from a hypertrophic lake sediment: Experiments with intact sediment cores in a continuous flow system. Arch. Hydrobiol., 116: 45-59.

SONZOGNI, W. C., D. M. JEFFS, J. C. KONRAD, J. B. ROBINSON, G. CHESTERS, D. R. COOTE \& R. C. OSTRY. 1980. Pollution from land runoff. Environ. Sci. Technol., 14: 148-153.

TARTARI, G. \& G. BIASCI. 1997. Trophic status and lake sedimentation fluxes. Water, Air and Soil Pollution, 99:523-531.

VOLLENWEIDER, R. A. 1968. Scientific fundamentals of the eutrophication of lakes and flowing waters, with particular reference to nitrogen and phosphorus as factors in eutrophication. Technical Report DAS/CSI/68.27, Environmental Directorate, Organization for Economic Cooperatation and Development (OECD), París. $154 \mathrm{p}$.

VOLLENWEIDER, R. A. 1976. Advances in defining critical loading levels for phosphorus in lake eutrophication. Mem. Ist. Ital. Idrobiol., 33:53-83.

WETZEL, R. G. 1975. Limnología. Barcelona: Omega. 